\title{
ON SYMPLECTIC FILLINGS OF LINKS OF RATIONAL SURFACE SINGULARITIES WITH REDUCED FUNDAMENTAL CYCLE
}

\author{
MOHAN BHUPAL ${ }^{\dagger}$
}

\begin{abstract}
We prove that every symplectic filling of the link of a rational surface singularity with reduced fundamental cycle admits a rational compactification, possibly after a modification of the filling in a collar neighbourhood of the link.
\end{abstract}

\section{$\S 1$. Introduction}

Let $O$ be an isolated singularity of an algebraic variety $V$. The link $L$ of $O \in V$ carries a natural contact structure $\xi$ given by the maximal complex subspaces of the tangent spaces of $L$. In this note we consider the case where $O$ is a rational surface singularity with reduced fundamental cycle. We prove the following.

THEOREM 1.1. Let $(L, \xi)$ be the link of a rational surface singularity $O \in V$ with reduced fundamental cycle. Then any symplectic filling $(W, \omega)$ of $(L, \xi)$ can be symplectically embedded into a rational symplectic 4-manifold $\left(X, \omega^{\prime}\right)$, after possibly modifying $W$ in a collar neighbourhood of $\partial W=L$.

The class of rational surface singularities with reduced fundamental cycle properly includes the class of cyclic quotient singularities. For the latter Theorem 1.1 follows from the work of McDuff [6] and Ohta and Ono [10] in special cases and Lisca [4] in the general case.

We prove Theorem 1.1 by constructing explicitly a concave symplectic filling $(Z, \sigma)$ of the link $(L, \xi)$ of the given rational surface singularity $O \in V$. By construction, our concave symplectic filling $(Z, \sigma)$ contains a rational symplectically embedded curve with self-intersection +1 . The proof

Received August 20, 2002.

2000 Mathematics Subject Classification: Primary: 53D99, Secondary: 32S25.

${ }^{\dagger}$ Supported by a JSPS Postdoctoral Fellowship For Foreign Researchers in Japan. 
of Theorem 1.1 is then completed by a gluing argument and then appealing to a theorem of McDuff [6].

It is interesting to ask whether or not the statement of Theorem 1.1 can be extended to the whole class of rational surface singularities. The methods used in this note do not extend beyond the class of rational surface singularities with reduced fundamental cycle although it is known that the statement of Theorem 1.1 does hold for certain examples of rational surface singularities without reduced fundamental cycle, for instance, the simple singularities $D_{n}, n \geq 4, E_{6}, E_{7}$ and $E_{8}$ (see [10]). For the class of singularities we consider one can see a positive rational curve in the compactification directly; in the other cases one can not directly see such a positive rational curve.

\section{§2. Preliminaries}

Let $\pi: \bar{V} \rightarrow V$ be the minimal resolution of a normal surface singularity $O \in V$. Then $O \in V$ is said to be a rational singularity if $R^{1} \pi_{*} \mathcal{O}_{\bar{V}}=0$ (see $[2])$. In particular, this implies that the exceptional divisor $E=\pi^{-1}(O)$ is a union $E=\bigcup E_{i}$ of rational curves. We may assume that the curves $E_{i}$ intersect transversely and are such that no three curves intersect in a point. Furthermore, we may assume that $E_{i}$ and $E_{j}$ intersect in at most one point for $i \neq j$. Let $\Gamma$ denote the dual resolution graph. By definition, this is the weighted graph that is obtained by taking one vertex $v_{i}$ for each irreducible component $E_{i}$ of $E$ and joining $v_{i}$ and $v_{j}$ with an edge whenever $E_{i}$ and $E_{j}$ intersect in a point. Each vertex $v_{i}$ of $\Gamma$ is weighted with the integer $n_{i}=E_{i} \cdot E_{i}$, the self-intersection of $E_{i}$. A further consequence of $O \in V$ being a rational singularity is that the dual resolution graph $\Gamma$ is a tree (see, for example, [8]). The fundamental cycle $Z$ is defined to be the smallest positive cycle $Z=\sum c_{i} E_{i}$ with $c_{i} \in \mathbb{Z}$ such that $Z \cdot E_{i} \leq 0$ for all $i$. The fundamental cycle $Z$ is said to be reduced if $Z=E$, that is, $c_{i}=1$ for all $i$. For each $i$, let $b_{i}$ be the number of rational curves $E_{j}, j \neq i$, such that $E_{i} \cdot E_{j} \neq 0$. Then $Z$ is reduced if and only if $-b_{i} \geq E_{i} \cdot E_{i}$ for all $i$. In particular, any weighted tree with sufficiently negative weights can be obtained as the dual resolution graph of some rational surface singularity with reduced fundamental cycle. Explicitly, any weighted linear graph, with all weights $\leq-2$, corresponds to the dual resolution graph of a rational surface singularity with reduced fundamental cycle. These correspond, of course, to the well-known cyclic quotient singularities $A_{n, q}$. 
Although the following discussion is valid for the wider class of normal surface singularities, for convenience, we restrict to the case of rational surface singularities. Given a rational surface singularity $O \in V$, choose an analytic embedding of a neighbourhood of $O$ in $V$ into $\mathbb{C}^{n}$ for some $n$ such that $O$ is mapped to the origin. The link $L$ of $O \in V$ is then defined to be the intersection of $V$ with the sphere $S^{2 n-1}(\varepsilon)$ of radius $\varepsilon$ for $\varepsilon$ sufficiently small. Now if we are given two rational surface singularities $O \in V$ and $O^{\prime} \in V^{\prime}$ with the same dual resolution graph, then by a theorem of Laufer [5] the analytic structure in a neighbourhood of the minimal resolution of $O \in V$ may be connected by a series of deformations to the analytic structure in a neighbourhood of the minimal resolution of $O^{\prime} \in V^{\prime}$. In particular, it follows from Gray's theorem that the contact structure on the link of $O \in V$ is isotopic to the contact structure on the link of $O^{\prime} \in V^{\prime}$. We may thus speak unambiguously of the contact structure on the link of a rational surface singularity having a given dual resolution graph.

Suppose that $(M, \xi)$ is a contact 3-manifold with $\xi=\operatorname{ker} \lambda$ for some 1-form $\lambda$ on $M$ defining the given co-orientation of $\xi$. A compact symplectic 4-manifold $(W, \omega)$ with $\partial W=M$ is said to be a (weak) symplectic filling of $(M, \xi)$ if the following two conditions hold.

(SF1) The orientation on $M$ given by $\lambda \wedge d \lambda$ coincides with the orientation on $M$ given as the boundary of $W$.

(SF2) $\left.\omega\right|_{\xi}$ is positively proportional to $\left.d \lambda\right|_{\xi}$.

Here $W$ is oriented by $\omega \wedge \omega$. If, furthermore, there exists an extension $\tilde{\lambda}$ of $\lambda$ to a collar neighbourhood of $M=\partial W$ such that $d \tilde{\lambda}=\omega$, then $(W, \omega)$ is said to be a strong symplectic filling of $(M, \xi)$. If we replace (SF1) by:

$\left(\mathrm{SF} 1^{\prime}\right)$ The orientation on $M$ given by $\lambda \wedge d \lambda$ is opposite to the orientation on $M$ given as the boundary of $W$,

we get the notions of weak and strong concave symplectic fillings.

In our case, that is when $(L, \xi)$ is the link of a rational surface singularity with reduced fundamental cycle with its canonical contact structure, we have that $L$ is a rational homology sphere. This follows from Mumford's computation of $\pi_{1}(L)$ (see [7]). In such a case it can be shown that every weak symplectic fillings $(W, \omega)$ of $(L, \xi)$ can be made into a strong symplectic filling after possibly modifying the symplectic structure in a collar neighbourhood $\partial W=L$ (see, for example, [3], [9]). For an explicit description of the contact structure $\xi$ see Page 56 below. 


\section{$\S 3$. Proof of Theorem 1.1}

Fix a rational surface singularity $O \in V$ with reduced fundamental cycle and denote by $\Gamma$ its dual resolution graph. Pick a vertex of $\Gamma$ which is connected by an edge to exactly one other vertex and label this vertex $v_{1}$. Suppose that $\Gamma$ has $m$ vertices. Now label the other vertices of $\Gamma v_{2}, \ldots, v_{m}$ by requiring that any path in $\Gamma$ starting at $v_{1}$ and ending at a leaf goes through vertices $v_{i_{1}}, \ldots, v_{i_{k}}$, in this order, where $i_{1}<\cdots<i_{k}$. Since $\Gamma$ is a tree, this is always possible.

Now consider the complex projective plane $\mathbb{C} P^{2}$ and fix a point $P \in$ $\mathbb{C} P^{2}$. Denote by $L_{\infty} \subset \mathbb{C} P^{2}$ any line which does not pass through $P$. We now describe an algorithm for successively blowing up $\mathbb{C} P^{2}$ at points away from the proper transform of $L_{\infty}$ so that in some blow-up of $\mathbb{C} P^{2}$ we obtain a configuration of rational curves which is described by the graph $\Gamma$.

To begin with, blow up $\mathbb{C} P^{2}$ at $P$ and denote the corresponding (-1)curve in the blow-up of $\mathbb{C} P^{2}$ by $D_{1}$. Denote the blow-up of $\mathbb{C} P^{2}$ at $P$ by $\mathbb{C} P_{1}^{2}$ and denote the proper transform of $L_{\infty}$ in $\mathbb{C} P_{1}^{2}$ by the same symbol. Now pick a point on $D_{1}$ and blow up $\mathbb{C} P_{1}^{2}$ at that point. Denote the resulting variety by $\mathbb{C} P_{2}^{2}$ and the corresponding $(-1)$-curve by $D_{2}$. Continue using the same notation to denote the proper transforms of $D_{1}$ and $L_{\infty} . D_{1}$ now becomes a (-2)-rational curve in $\mathbb{C} P_{2}^{2}$. If necessary, now blow up $\mathbb{C} P_{2}^{2}$ at $-2-n_{1}$ points along $D_{1} \backslash D_{2}$, where $n_{1}=\operatorname{wt}\left(v_{1}\right)$, the weight of $v_{1}$, and denote the resulting variety by $\mathbb{C} P_{k_{1}}^{2}$, where $k_{1}=-n_{1}$, the number of times we have successively blown up $\mathbb{C} P^{2}$. Then in $\mathbb{C} P_{k_{1}}^{2}, D_{1}$ will be a rational curve with self-intersection $n_{1}$ and $D_{2}$ will be a rational curve with self-intersection -1 intersecting $D_{1}$ once.

Now suppose that curves $D_{1}, \ldots, D_{l-1}$ have been obtained in some variety $\mathbb{C} P_{k_{l-1}}^{2}$, obtained by successively blowing up $\mathbb{C} P^{2}$, and that $D_{i} \cdot D_{i}=$ $n_{i}=\operatorname{wt}\left(v_{i}\right)$ for $i=1, \ldots l-1$ and, for $1 \leq i<j \leq l-1, D_{i}$ intersects $D_{j}$ in one point if and only if $v_{i}$ is connected to $v_{j}$ by an edge. If $l-1=m$ we are done, otherwise $D_{l}$ will be some $(-1)$-curve in $\mathbb{C} P_{k_{l-1}}^{2}$ which was obtained by some previous blow up. By the induction hypothesis, $D_{l}$ will intersect precisely one curve $D_{l^{\prime}}$ for $1 \leq l^{\prime} \leq l-1$, where $l^{\prime}$ is the index of the unique vertex $v_{l^{\prime}}$ such that $l^{\prime} \leq l-1$ and $v_{l}$ and $v_{l^{\prime}}$ are connected by an edge. Now let

$$
d_{l}=\#\left\{v_{j} \mid v_{j} \text { is connected by an edge to } v_{l}, j>l\right\} .
$$

Assuming that $d_{l} \neq 0$, now blow up $\mathbb{C} P_{k_{l-1}}^{2}$ at $d_{l}$ points along $D_{l} \backslash D_{l^{\prime}}$. Denote the blown-up variety by $\mathbb{C} P_{k}^{2}$, where $k=k_{l-1}+d_{l}$, and the corre- 
sponding (-1)-rational curves by $D_{j_{1}}, \ldots, D_{j_{d_{l}}}$. Here $j_{1}, \ldots j_{d_{l}}$ denote the indices of the $v_{j}, j>l$, such that $v_{j}$ is connected by an edge to $v_{l}$. Then $D_{l} \subset \mathbb{C} P_{k}^{2}$ will have self-intersection $-\left(d_{l}+1\right)$. By the reduced condition $-\left(d_{l}+1\right) \geq n_{l}=\operatorname{wt}\left(v_{l}\right)$. If necessary, now blow up $\mathbb{C} P_{k}^{2}$ at $-\left(d_{l}+1\right)-n_{l}$ points along $D_{l} \backslash\left(D_{l^{\prime}} \cup D_{j_{1}} \cup \cdots \cup D_{j_{d_{2}}}\right)$ and denote the blown-up variety by $\mathbb{C} P_{k_{l}}^{2}$, where $k_{l}=k-1-n_{l}$. Then, in $\mathbb{C} P_{k_{l}}^{2}, D_{l}$ will have self-intersection $n_{l}$. This completes the inductive step.

Following the above algorithm we obtain a variety $\mathbb{C} P_{N}^{2}$ containing a bunch of rational curves $D_{1}, \ldots, D_{m}$ intersecting in the manner described by $\Gamma$ and having self-intersections $D_{i} \cdot D_{i}=n_{i}=\operatorname{wt}\left(v_{i}\right) . \mathbb{C} P_{N}^{2}$ also contains a rational curve $L_{\infty}$ which has self-intersection +1 and is disjoint from each of the curves $D_{i}$. Now note, by the Segre embedding, that $\mathbb{C} P_{N}^{2}$ is a projective variety. Moreover, by Artin's theorem on contractibility of curves on algebraic surfaces [1], it follows that after contracting $D=D_{1} \cup \cdots \cup D_{m}$ the surface we obtain is again projective. Denote this surface by $V^{\prime} \subset \mathbb{C} P^{n}$ and let $Q \in V^{\prime}$ denote the image of $D$ under the contraction. By applying a projective linear transformation if necessary we may assume that $Q=[1$ : $0: \cdots: 0]$. We now show that the complement of a neighbourhood of $Q$ in $V^{\prime}$ is a strong concave filling of the link of our rational singularity $O$ with reduced fundamental cycle. ${ }^{1}$

Let $\left[Z_{0}: \ldots: Z_{n}\right]$ denote homogeneous coordinates on $\mathbb{C} P^{n}$ and let $z_{i}=Z_{i} / Z_{0}, i=1, \ldots, n$ denote complex coordinates on the open set $U_{0}=$ $\left(Z_{0} \neq 0\right)$ in $\mathbb{C} P^{n}$. Then on $U_{0}=\mathbb{C}^{n}$, the restriction of the Fubini-Study metric is given by

$$
\left.\omega_{F S}\right|_{\mathbb{C}^{n}}=\frac{i}{2}\left(\frac{\sum_{j=1}^{n} d z_{j} \wedge d \bar{z}_{j}}{1+\sum_{j=1}^{n} z_{j} \bar{z}_{j}}-\frac{\left(\sum_{j=1}^{n} \bar{z}_{j} d z_{j}\right) \wedge\left(\sum_{j=1}^{n} z_{j} d \bar{z}_{j}\right)}{\left(1+\sum_{j=1}^{n} z_{j} \bar{z}_{j}\right)^{2}}\right) .
$$

Now note that $\left.\omega_{F S}\right|_{\mathbb{C}^{n}}=d \lambda_{0}$, where $\lambda_{0}$ is the 1 -form on $\mathbb{C}^{n}$ given by

$$
\lambda_{0}=\frac{i}{4}\left(\frac{\sum_{j=1}^{n}\left(z_{j} d \bar{z}_{j}-\bar{z}_{j} d z_{j}\right)}{1+\sum_{j=1}^{n} z_{j} \bar{z}_{j}}\right) .
$$

It follows that $\operatorname{ker}\left(\left.\lambda_{0}\right|_{S^{2 n-1}(\varepsilon)}\right)$ consists precisely of those vectors $X \in T_{z} S^{2 n-1}(\varepsilon)$ such that $J_{0} X$ is also in $T_{z} S^{2 n-1}(\varepsilon)$, where $J_{0}$ is the standard complex

\footnotetext{
${ }^{1}$ This fact was already known to Ono.
} 
structure on $\mathbb{C}^{n}$. In other words, $\operatorname{ker}\left(\left.\lambda_{0}\right|_{S^{2 n-1}(\varepsilon)}\right)$ gives the standard contact structure on $T S^{2 n-1}(\varepsilon)$. Let $L=V^{\prime} \cap S^{2 n-1}(\varepsilon)$ denote the link of $Q \in V^{\prime}$. It follows easily that $\operatorname{ker}\left(\left.\lambda_{0}\right|_{L}\right)$ gives the natural contact structure $\xi$ on $L$ and hence that the intersection of $V^{\prime}$ with the ball $B^{2 n}(\varepsilon)$ of radius $\varepsilon$ gives a strong symplectic filling of the $\operatorname{link}(L, \xi)$ of $Q \in V^{\prime}$. Hence the complement of $V^{\prime} \cap B^{2 n}(\varepsilon)$ in $V^{\prime}$ gives a strong concave filling, $(Z, \sigma)$, of $(L, \xi)$ as required.

Now suppose that $(W, \omega)$ is a symplectic filling of the link $(L, \xi)$ of $O \in V$. Then, as noted before, the symplectic structure $\omega$ can be modified into another symplectic structure $\omega^{\prime}$ such that $\left(W, \omega^{\prime}\right)$ is a strong symplectic filling of $(L, \xi)$. We can now symplectically embed a collar neighbourhood of $\partial W \subset(W, \omega)$ into the symplectization of $(L, \xi)$ such that the outward pointing normal vector along $\partial W$ points in the positive direction of the symplectization. Similarly, we can symplectically embed collar neighbourhood of $\partial Z \subset(Z, \sigma)$ into the symplectization of $(L, \xi)$ such that the outward pointing normal vector along $\partial Z$ points in the negative direction of the symplectization. Thus, after possibly rescaling the symplectic structure $\sigma$, we obtain a closed symplectic 4 -manifold $\left(X, \omega^{\prime \prime}\right)$ containing a symplectically embedded copy of $\left(W, \omega^{\prime}\right)$. By construction, $\left(X, \omega^{\prime \prime}\right)$ contains a symplectically embedded rational curve of self-intersection +1 . A theorem of McDuff [6] now shows that $\left(X, \omega^{\prime \prime}\right)$ is symplectomorphic to $\mathbb{C} P^{2}$ blown up at a finite number of points. This completes the proof of Theorem 1.1.

Acknowledgements. I wish to thank Prof. K. Ono for helpful discussions.

\section{REFERENCES}

[1] M. Artin, Some numerical criteria for contractibility of curves on algebraic surfaces, Amer. J. Math., 84 (1962), 485-496.

[2] - On isolated rational singularities of surfaces, Amer. J. Math., 88 (1966), $129-136$.

[3] Y. Eliashberg, On symplectic manifolds with some contact properties, J. Diff. Geom., 33 (1991), 233-238.

[4] P. Lisca, On lens spaces and their symplectic fillings, preprint.

[5] H. B. Laufer, Deformations of resolutions of two-dimensional singularities, Geometry of singularities (Proc. Conf., Rice Univ., Houston, Tex., 1972). Rice Univ. Studies, 59 (1973), no. 1, 53-96.

[6] M. McDuff, The structure of rational and ruled symplectic 4-manifolds, J. Amer. Math. Soc., 3 (1990), 679-712; [erratum], 5 (1992), 987-8. 
[7] D. Mumford, The topology of normal singularities of an algebraic surface and a criterion for simplicity, IHES 1961, Publ. Math. No. 9 (1961), 5-22.

[8] A. Némethi, Five lectures on normal surface singularities, Bolyai Soc. Math. Stud., 8, Low dimensional topology (Eger, 1996/Budapest, 1998), 269-351, János Bolyai Math. Soc., Budapest, 1999.

[9] H. Ohta and K. Ono, Simple singularities and topology of symplectically filling 4-manifolds, Comment. Math. Helv., 74 (1999), 575-590.

[10] _ Simple singularities and symplectic fillings, preprint.

Department of Mathematics,

Hokkaido University

Sapporo 060-0810

Japan

bhupal@math.sci.hokudai.ac.jp 